PAEDIATRIC LUNG DISEASE

\title{
Frequent use of chemical household products is associated with persistent wheezing in pre-school age children
}

\author{
A Sherriff, A Farrow, J Golding, the ALSPAC Study Team, J Henderson
}

Thorax 2005;60:45-49. doi: 10.1136/thx.2004.021154

See end of article for authors' affiliations

.....................

Correspondence to: $\operatorname{Dr}$ A Sherriff, Unit of Paediatric and Perinatal Epidemiology, Division of Child Health, University of Bristol, Bristol BS8 1BR, UK; Andrea.Sherriff@bris. ac.uk

Received 7 January 2004 Accepted 20 September 2004
Background: In the UK and other developed countries the prevalence of asthma symptoms has increased in recent years. This is likely to be the result of increased exposure to environmental factors. A study was undertaken to investigate the association between maternal use of chemical based products in the prenatal period and patterns of wheeze in early childhood.

Methods: In the population based Avon Longitudinal Study of Parents and Children (ALSPAC), the frequency of use of 11 chemical based domestic products was determined from questionnaires completed by women during pregnancy and a total chemical burden (TCB) score was derived. Four mutually exclusive wheezing patterns were defined for the period from birth to 42 months based on parental questionnaire responses (never wheezed, transient early wheeze, persistent wheeze, and late onset wheeze). Multinomial logistic regression models were used to assess the relationship between these wheezing outcomes and TCB exposure while accounting for numerous potential confounding variables. Complete data for analysis was available for 7019 of 13971 (50\%) children.

Results: The mean (SD) TCB score was 9.4 (4.1), range 0-30. Increased use of domestic chemical based products was associated with persistent wheezing during early childhood (adjusted odds ratio (OR) per unit increase of TCB 1.06 (95\% confidence interval (Cl) 1.03 to 1.09)) but not with transient early wheeze or late onset wheeze. Children whose mothers had high TCB scores (>90th centile) were more than twice as likely to wheeze persistently throughout early childhood than children whose mothers had a low TCB score (<10th centile) (adjusted OR 2.3 (95\% Cl 1.2 to 4.4)).

Conclusion: These findings suggest that frequent use of chemical based products in the prenatal period is associated with persistent wheezing in young children. Follow up of this cohort is underway to determine whether TCB is associated with wheezing, asthma, and atopy at later stages in childhood.

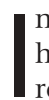
$\mathrm{n}$ the UK the prevalence of asthma symptoms in children has increased in recent years, ${ }^{1-5}$ a trend which has been reflected in numerous developed countries worldwide. ${ }^{6-9}$ This is most probably due to a change in some factor or factors in the environment which, if identified, might lead to remediation and even prevention of the disease in the future. ${ }^{10}$ It has been postulated that the indoor air environment may play an important role in the increasing asthma problem due to the fact that people (especially mothers with young children) spend most of the day indoors. ${ }^{11}$

In some susceptible individuals the development of respiratory hypersensitivity or asthmatic symptoms has been associated with indiscriminate use of household products and exposure to specific compounds such as the diisocyanates, organic acid anhydrides, styrene and hydroquinone, and formaldehyde. ${ }^{12}$ Formaldehyde, a constituent of a large number of products owing to its bactericidal action, is ubiquitous in indoor environments. It is found in cleaning chemicals and cosmetics but also carpets, wallpaper and furniture. A Louisiana indoor air quality survey reported on levels of airborne formaldehyde where approximately $60 \%$ exceeded the American Society of Heating, Refrigeration and Air Conditioning Engineers guideline of $0.123 \mathrm{mg} / \mathrm{m}^{3}$. A high level of formaldehyde was suggested as a potential upper respiratory irritant. ${ }^{13}$ A small school study also found that, among children without a history of atopy, a new asthma diagnosis was more common where higher concentrations of formaldehyde and total moulds were found in classroom air. ${ }^{14}$ Data from community and occupational studies have consistently shown formaldehyde in association with allergic respiratory response and both acute and chronic health problems. ${ }^{15}$

There has been a great deal of research into the adverse respiratory health effects of chemical agents within occupational settings. Occupation defined by job title and asthma data assessed across 12 industrialised countries indicated the highest statistically significant risk of asthma to be among farmers (odds ratio (OR) 2.62; 95\% CI 1.29 to 5.35) and cleaners (OR $1.97 ; 95 \%$ CI 1.33 to 2.92 ). It has also been suggested that the prevalence of occupational asthma in women in specific occupations is underestimated..$^{16}$ Other studies throughout Europe and the USA have found an increased risk of asthma (and asthma related symptoms) in individuals working as cleaners. ${ }^{17-22}$

Other epidemiological evidence gives support to an association between individual constituents of chemical household products such as volatile organic compounds (mixed and single constituents), ${ }^{23}$ formaldehyde, ${ }^{24}$ and fragrances ${ }^{25} 26$ and the risk of adverse respiratory symptoms. However, some evidence is contradictory because of uncertain exposure assessment and symptom registration, inadequate sample sizes, and inappropriate study designs. ${ }^{10}$

Despite a large body of occupational literature, there are virtually no data available at the population level on the effect of domestic use of chemical based products on the respiratory health of young children. The aim of this study was therefore to examine the effect of prenatal exposure to multiple chemical agents on patterns of wheeze (never wheezed, transient early wheeze, persistent wheeze, late onset wheeze) during the first 3.5 years in children taking 
part in the Avon Longitudinal Study of Parents and Children (ALSPAC). ${ }^{27} 28$

\section{METHODS}

\section{Study sample}

The ALSPAC is a prospective study of 14541 pregnancies that resulted in 13971 live births surviving to 1 year. Women were enrolled as early in pregnancy as possible on the basis of an expected date of delivery between 1 April 1991 and 31 December 1992 and place of residence within the three Bristol based health districts of the former county of Avon, UK. Ethical approval was obtained from the medical ethical committees of the three Avon health districts. It was estimated that $85-90 \%$ of eligible mothers were enrolled in the study. Data were collected from questionnaires completed by the parents, medical records, and biological samples. The study mothers completed four questionnaires during pregnancy. Questionnaires relating to the child were administered at $1,6,15,18,24,30$ months and at 6 monthly intervals thereafter, and to the study mothers and their partners on an annual basis.

\section{Data collection \\ Wheezing}

Questionnaires on wheezing patterns of the study child were completed by a parent or primary carer (usually the mother) at $6,18,30$, and 42 months after birth. Each mother was asked whether her child had experienced wheezing with whistling on his/her chest during each of the study periods: 0-6 months; 6-18 months; $18-30$ months; and 3042 months. This information was used to identify four mutually exclusive patterns of wheeze between birth and 3.5 years: never wheezed (no wheezing at any of the four time points); transient early wheeze (wheezed 0-6 months but not thereafter); persistent wheeze (wheezed during each of the periods 6-18 months, 18-30 months, and 3042 months); late onset wheeze (onset of wheeze between 30 and 42 months).

\section{Total chemical burden (TCB)}

During the pregnancy the study women were asked to complete a questionnaire on certain aspects of their health, environment, and lifestyle. In particular, they were asked to answer the question: "During this pregnancy how often have you used the following (a list of 15 chemical based products)?" Available responses were: not at all, less than once a week, about once a week, most days, every day. Fifteen product categories were included in the questionnaire and, from this initial list, we selected the 11 most frequently used (by at least $5 \%$ of the study sample). The products chosen (and the percentages of women using them) were: disinfectant $(87.4 \%)$, bleach $(84.8 \%)$, carpet cleaner $(35.8 \%)$, window cleaner $(60.5 \%)$, dry cleaning fluid $(5.4 \%)$, aerosols $(71.7 \%)$, turpentine/white spirit $(22.6 \%)$, air fresheners (spray, stick or aerosol) (68\%), paint stripper (5.5\%), paint or varnish $(32.9 \%)$, and pesticides/insect killers (21.2\%). A simple score for frequency of use of each product was derived $(0=$ not at all, $\mathrm{l}=$ less than once a week, $2=$ about once a week, $3=$ most days, $4=$ every day) and the scores for each product were summed to produce a total chemical burden (TCB) score for each respondent which could range from 0 (no exposure) to 55 (exposed to all 11 products daily).

\section{Potential confounding variables}

A number of variables were considered as potential confounders of the association between wheezing and TCB. These included exposure to environmental tobacco smoke (ETS), maternal smoking during pregnancy, maternal history of asthma, maternal parity, crowding in the home, sex of the

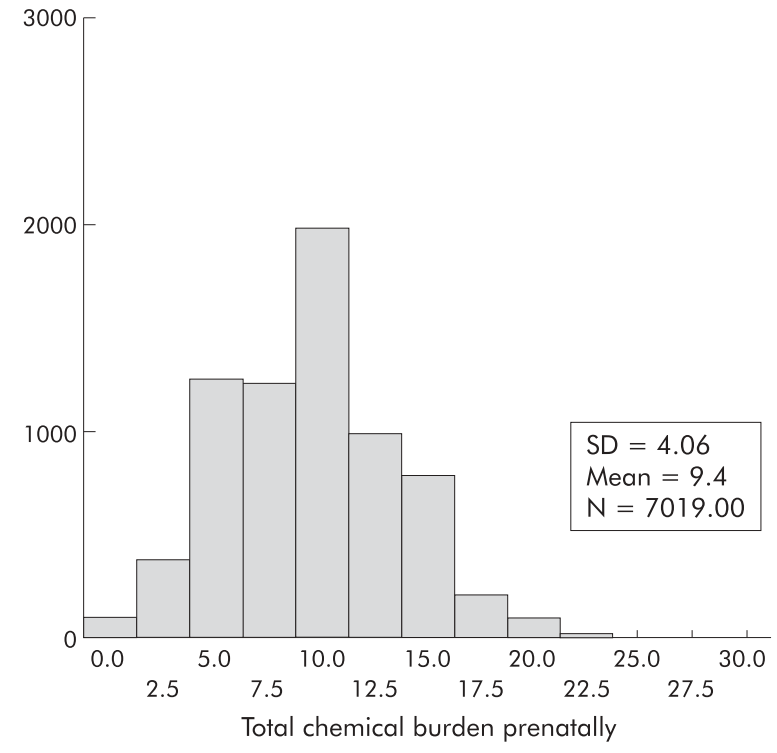

Figure 1 Distribution of total chemical burden (TCB) score during pregnancy.

study child, contact with domestic pets, damp housing, maternal age at delivery, highest education level of mother, housing tenure, duration of breastfeeding, number of hours mother worked outside home, and season of return of chemical use questionnaire. This information was gathered from self-report questionnaires and birth records.

\section{Statistical methods}

All analyses were undertaken using SPSS for Windows (version 9.0). Multinomial logistic regression models were used to analyse associations between TCB score and patterns of wheeze using the never wheezed group as the reference category. Separate models were produced using the TCB score as a continuous factor and as a dichotomous factor $(<10$ th centile versus $>90$ th centile). Unadjusted and adjusted odds ratios (ORs) and 95\% confidence intervals (CIs) were calculated for all patterns of wheeze compared with the never wheezed group.

To assess whether a linear relationship with each wheezing pattern was appropriate, the TCB score was fitted as a continuous factor in a model and then as a categorical factor. For the categorical factor the TCB score was split into deciles (there were only 28 distinct TCB scores so 10 groups provided adequate numbers per group). The likelihood ratio test statistic was used to determine whether there was significant departure from linearity in the relationship between TCB score and wheezing patterns. For each wheezing pattern there was no evidence against the null hypothesis that the effect of the TCB score is linear (transient early wheeze: $\mathrm{p}>0.1$; persistent wheeze: $\mathrm{p}>0.1$; late onset wheeze: $\mathrm{p}>0.1$ ).

Finally, to determine whether a single product was responsible for any observed effect, the TCB score was recalculated 11 times by sequential removal of each individual product category followed by repeat analysis of the score containing the remaining 10 products.

\section{RESULTS}

Of the 13971 ALSPAC children alive at 1 year, 8134 children had complete data for exposure (TCB score) and had responded to all four wheezing questionnaires. However, based on these responses, 7019 children $(50 \%)$ could then be categorised into one of the four mutually exclusive wheezing pattern groups (the remainder did not fall into a homogenous 
Table 1 Unadjusted and adjusted odds ratios (ORs) and $95 \%$ confidence intervals (Cls) for wheezing phenotypes* (transient early wheeze, persistent wheeze, and late onset wheeze (0-42 months)) according to total chemical burden (TCB) score measured during pregnancy (continuous)

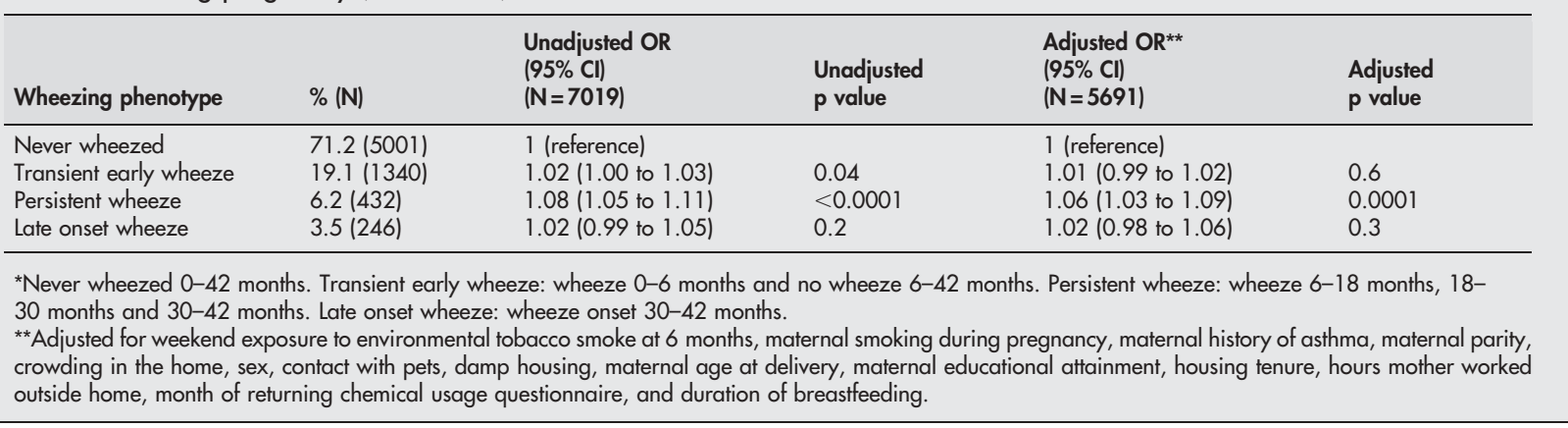

group and were therefore excluded from the present analysis). Between birth and 3.5 years, $71.2 \%$ of children $(\mathrm{n}=5001)$ never wheezed, $19.1 \%(\mathrm{n}=1340)$ had transient early wheeze, $6.2 \%(\mathrm{n}=432)$ had persistent wheeze, and $3.5 \%(n=246)$ had late onset wheeze.

Figure 1 shows the distribution of the TCB score in the prenatal period. From the sample analysed, the score ranged between 0 and 30 and is approximately normally distributed with a mean (SD) of $9.4(4.1)$.

Table 1 presents unadjusted and adjusted odds ratios (with $95 \%$ CIs) for patterns of wheeze (0-42 months) according to a continuous TCB score. In the unadjusted models increasing TCB score was significantly associated with persistent wheeze and transient early wheeze but not with late onset wheeze. After adjustment, however, only persistent wheeze remained statistically significant.

Persistent wheeze was more than twice as likely to occur in children of women in the top decile of TCB score than in children of women in the bottom decile (adjusted OR 2.3 (95\% CI 1.2 to 4.4 ), table 2 ). A similar but non-significant effect was observed for late onset wheeze (adjusted OR 2.0 (95\% CI 0.8 to 5.2$)$ ) but the proportion of children with transient early wheeze did not differ significantly when top and bottom deciles of maternal TCB score were compared.

All analyses were repeated 11 times, each time removing one of the chemicals from the score. In all cases there was no significant change in effect sizes, which suggests that no single product was implicated in the association with infant wheezing (data available on request).

\section{DISCUSSION}

A dose-dependent relationship was observed between frequency of use of common household chemical products in the prenatal period and persistent wheeze in the resulting offspring. This finding was independent of numerous potential confounding factors. Late onset wheeze may be more likely in children whose mothers had a very high TCB score ( $>90$ th centile). Transient early wheeze was not significantly associated with TCB score.

This was a large population based longitudinal study which collected exposure data during the study pregnancy and outcome data after delivery, and was able to consider a large number of potential confounding variables within the statistical analyses.

It was not possible to determine whether the observed effect was due to in utero exposure or whether prenatal exposure was a proxy measure for general chemical use postnatally. In a post hoc analysis we explored the relationship between postnatal TCB exposure (at 8 months post partum) and wheezing phenotypes and found that, not only were TCB scores correlated prenatally and postnatally, but there was also a similar relationship between TCB score (continuous and dichotomous) and persistent wheeze (although the magnitude of the effect was smaller postnatally). Transient early wheeze and late onset wheeze were not associated with postnatal TCB.

An assumption of our study was that exposure in utero came primarily from the women's use of chemicals, and there was no attempt to quantify use in the proximity of the pregnant woman by other household members during the study pregnancy. This potentially could underestimate the in utero exposure of the fetus, however use by other household members would have to be greatly different or in the opposite direction to that of the pregnant women for the direction of effect to be altered significantly.

We were also able to rule out the possibility that women with a history of asthma modified their exposure as a result of their condition (either stopped using chemicals or used

Table 2 Unadjusted and adjusted odds ratios (ORs) and 95\% confidence intervals (Cls) for wheezing phenotypes* (transient early wheeze, persistent wheeze, and late onset wheeze (0-42 months)) according to total chemical burden (TCB) score measured during pregnancy (bottom decile versus top decile)

\begin{tabular}{|c|c|c|c|c|c|c|}
\hline Wheezing phenotype & $\begin{array}{l}\text { Bottom decile } \\
\text { of TCB } \\
\% \text { (N) }\end{array}$ & $\begin{array}{l}\text { Top decile } \\
\text { of TCB } \\
\%(N)\end{array}$ & $\begin{array}{l}\text { Unadjusted OR } \\
(95 \% \mathrm{Cl}) \\
(\mathrm{N}=7019)\end{array}$ & $\begin{array}{l}\text { Unadjusted } \\
\text { p value }\end{array}$ & $\begin{array}{l}\text { Adjusted OR }{ }^{* *} \\
(95 \% \mathrm{Cl}) \\
(\mathrm{N}=5691)\end{array}$ & $\begin{array}{l}\text { Adjusted } \\
\text { p value }\end{array}$ \\
\hline Never wheezed & 74.9 (603) & 66.9 (338) & 1 (reference) & & 1 (reference) & \\
\hline Transient early wheeze & $18.8(151)$ & $19.0(96)$ & $1.13(0.90$ to 1.50$)$ & 0.4 & $0.94(0.60$ to 1.40$)$ & 0.7 \\
\hline Persistent wheeze & 4.0 (32) & $10.1(51)$ & 2.84 (1.79 to 4.51$)$ & $<0.0001$ & 2.30 (1.20 to 4.39$)$ & 0.012 \\
\hline Late onset wheeze & $2.4(19)$ & $4.0(20)$ & 1.88 (0.99 to 3.57$)$ & 0.05 & $2.02(0.80$ to 5.15$)$ & 0.14 \\
\hline
\end{tabular}

*Never wheezed 0-42 months. Transient early wheeze: wheeze 0-6 months and no wheeze 6-42 months. Persistent wheeze: wheeze 6-18 months, 1830 months and 30-42 months. Late onset wheeze: wheeze onset 30-42 months.

**Adjusted for weekend exposure to environmental tobacco smoke at 6 months, maternal smoking during pregnancy, maternal history of asthma, maternal parity, crowding in the home, sex, contact with pets, damp housing, maternal age at delivery, maternal educational attainment, housing tenure, hours mother worked outside home, month of returning chemical usage questionnaire, and duration of breastfeeding. 
more for a cleaner environment). TCB exposure in women with asthma and women without asthma were similar (11\% of women in the lowest decile of TCB had reported a history of asthma compared with $10.9 \%$ of women in the top decile of TCB score, $\mathrm{p}=0.2$ ).

This study did not attempt to identify and analyse individual chemical agents used in the home environment with respect to childhood wheezing. The primary interest was to establish whether cumulative exposure to domestic cleaning products had any adverse effect on the wheezing patterns of the child. An analogous approach has been used in studies of the effects of particulate air pollution on respiratory health in which the exposure variable comprises a composite measure of several different components, and has been shown in many studies to be associated with adverse respiratory health outcomes. However, the particular components associated with the outcome remain unknown. We attempted to evaluate whether a single product category may have dominated the effect by removal of each product sequentially from the analysis but no significant change in effect size was observed. However, it is acknowledged that some chemical constituents of these products may appear in more than one category, thus limiting the ability of this approach to identify individual chemicals that may have been associated with the outcome.

It is acknowledged that the interpretation of the odds ratios based on TCB score as a continuous factor is somewhat complicated due to the ordinal nature of the categories used to derive the score. As an epidemiological study, this study was not designed to identify and quantify the effect of individual chemical constituents on wheeze in an attempt to advise on exposure reduction methods; more detailed studies are required to follow on from this. Nonetheless, we were able to show a clear and strong effect of increased usage on persistent wheezing.

Although there were no objective measurements of indoor air exposures in this study, an earlier validation study on a subset of the ALSPAC population $(n=170)$ found a positive association between total levels of volatile organic compounds and self-reported use of air fresheners and aerosols, and these in turn were associated with maternal and infant symptoms. ${ }^{29}$

ALSPAC is a geographically based population cohort broadly representative of the population of Great Britain (1991 census) in terms of demographic, lifestyle, and health factors. $^{27}{ }^{28}$ Of the 13971 ALSPAC children alive at 1 year, 8134 had complete data for exposure (TCB score) and had responded to all four wheezing questionnaires. However, based on these responses, $7019(50 \%)$ children could then be categorised into one of the four mutually exclusive wheezing pattern groups ( the remainder did not fall into a homogenous group and were therefore excluded from the present analysis). Perhaps not surprisingly, there was some selection bias among those who had complete data: this group had significantly better educated older mothers living in owner occupied housing with fewer non-white children who weighed more at birth. In addition, those excluded from the analysis due to lack of chemical use data were more likely to have wheezed at all ages than those included, and those excluded from the analysis due to missing symptom data had, on average, higher TCB scores than those in the analysis.

To date, there has been little research on prenatal exposure to chemical agents and its effect on early childhood wheezing. A recent study examining the effects of direct exposure to volatile organic compounds of children aged 9-11 years found no association with wheezing illness ${ }^{30}$ but, by this age, the majority of wheezing illness is likely to be established asthma and this may have a different aetiology to wheezing illnesses that develop in early childhood. Wheezing before 3 years of age has been shown to be associated with abnormal airway function that persists in later childhood. ${ }^{31}$ Diminished lung function before the onset of symptoms in the postnatal period ${ }^{32}$ and wheeze in early infancy ${ }^{33}$ have both been observed to be associated with prenatal exposure to tobacco smoke.

This epidemiological study has shown a statistically strong association between prenatal exposure to domestic chemical products and persistent wheezing illness in young children up to the age of 3.5 years. When comparing the highest with the lowest decile of exposures, a similar but statistically nonsignificant effect was also observed for late onset wheeze, whereas transient early wheezing showed no association. There are epidemiological similarities in the risk factors associated with persistent and late onset wheezing that suggest these conditions share pathophysiological features ${ }^{34}$ consistent with asthma, while transient early wheezing is believed to represent a developmental airway abnormality that results in wheezing with viral respiratory infections. ${ }^{31}$ The positive associations between chemical exposures and wheezing phenotypes in the present study therefore support an effect on the development of airway inflammation and asthma rather than a fundamental effect on airway development in utero. Given the strong correlation between prenatal and postnatal use of household cleaning products in this population and their association with persistent wheezing, it is conceivable that this represents postnatal exposure with a direct inflammatory insult to the airways rather than a prenatal "priming" of airway inflammation in response to postnatal exposures such as airborne allergens. However, the causal mechanisms underlying the observed relationships remain to be determined. It is interesting to note that the prevalence of so-called "late onset wheezing" was relatively low in the present study. The occurrence of incident cases after the age of 3.5 years will increase the prevalence of this phenotype and it will be interesting to note if a positive association with early chemical exposures becomes apparent at later time points. Further research is needed to establish what, if any, are the casual mechanisms underlying such associations and what specific components of household chemical exposure are involved.

In conclusion, these findings suggest that children whose mothers make frequent use of chemical-based domestic products during pregnancy are more likely to wheeze persistently throughout early childhood, independent of many other indoor air exposures and other potential confounding factors.

\section{ACKNOWLEDGEMENTS}

The authors thank all the mothers who took part in the study and the midwives for their cooperation and help in recruitment. The whole ALSPAC study team comprises interviewers, computer technicians, laboratory technicians, clerical workers, research scientists, volunteers and managers who continue to make the study possible. The ALSPAC study is part of the WHO initiated European Longitudinal Study of Pregnancy and Childhood. The authors also thank Dr Sutapa Mukherjee and Professor David Strachan for their helpful comments during the preparation of this manuscript.

\section{Authors' affiliations \\ A Sherriff, J Golding, The ALSPAC Study Team, J Henderson, Unit of Paediatric and Perinatal Epidemiology, Division of Child Health, University of Bristol, Bristol BS8 1BR, UK \\ A Farrow, Department of Health and Social Care, Brunel University, Isleworth, Middlesex TW7 5DU, UK}

This study was funded by the Medical Research Council, the Wellcome Trust, the Medical Research Council, the University of Bristol, the Department of Health, and the Department of the Environment.

Competing interests: none. 


\section{REFERENCES}

1 Ninan TK, Russell G. Respiratory symptoms and atopy in Aberdeen schoolchildren: evidence from two survey 25 years apart. BMJ 1992:304:873-5

2 Anderson HR, Butland BK, Strachan DP. Trends in prevalence and severity of childhood asthma. BMJ 1994;308:1600-4.

3 Burr ML, Butland BK, King S, et al. Changes in asthma prevalence: two surveys 15 years apart. Arch Dis Child 1989;64:1452-6.

4 Lewis S, Butland BK, Strachan DP, et al. Study of the aetiology of wheezing illness at age 16 in two national British birth cohorts. Thorax 1996;51:670-6.

5 Omran M, Russell G. Continuing increase in respiratory symptoms and atopy in Aberdeen schoolchildren. BMJ 1996;3:12-34.

6 Murphy JF. Asthma: increasing despite advances in therapy. Ir Med J 1998;91:76.

7 Gerstman BB, Bosco LA, Tomita DK. Trends in the prevalence of asthma hospitalisation in the 5 to 14 years old Michigan Medicare population, 19801986. Pediatrics 1993:81:1-7.

8 Peat JK, van der Berg RH, Green WK, et al. Changing prevalence of asthma in Australian children. BMJ 1994;308:1591-6.

9 Sly RM. Changes in prevalence of allergic rhinitis and asthma. Ann Allergy Asthma Immunol 1999:82:233-48.

10 Institute of Medicine. Clearing the air: asthma and indoor air exposures. Washington, DC: National Academy Press, 2000.

11 Farrow A, Taylor H, Golding J. Time spent in the home by different family members. Environ Technol 1997;18:605-14.

12 Becher R, Hongslo JK, Jantunen MJ, et al. Environmental chemicals relevant for respiratory hypersensitivity: the indoor environment. Toxicol Lett 1996;86:155-62.

13 Lemus R, Abdelghani AA, Akers TG, et al. Potential health risks from exposure to indoor formaldehyde. Rev Environ Health 1998;13:91-8.

14 Smedje G, Norback D. Incidence of asthma diagnosis and self-reported allergy in relation to the school environment--a four-year follow-up study in schoolchildren. Int J Tuberc Lung Dis 2001;5:1059-66.

15 Delfino RJ. Epidemiologic evidence for asthma and exposure to air toxics: linkages between occupational, indoor, and community air pollution research. Environ Health Perspect 2002;110(Suppl 4):573-89.

16 Kogevinas M, Anto JM, Sunyer J, et al. Occupational asthma in Europe and other industrialised areas: a population-based study. European Community Respiratory Health Survey Study Group. Lancet 1999;353:1750-4.

17 Nielsen J, Bach E. Work-related eye symptoms and respiratory symptoms in female cleaners. Occup Med 1999;49:291-7.

18 Zock JP, Kogevinas M, Sunyer J, et al. Asthma risk, cleaning activities and use of specific cleaning products among Spanish indoor cleaners. Scand J Work Environ Health 2001;27:76-81
19 Karjalainen A, Martikainen R, Karjalainen J, et al. Excess incidence of asthma among Finnish cleaners employed in different industries. Eur Respir $J$ 2002;19:90-5.

20 Zock JP, Kogevinas M, Sunyer J, for the European Community Respiratory Health Survey, et al. Asthma characteristics in cleaning workers, workers in other risk jobs and office workers. Eur Respir J 2002;20:679-85.

21 Arif AA, Whitehead LW, Delclos GL, et al. Prevalence and risk factors of work related asthma by industry among United States workers: data from the third national health and nutrition examination survey (1988-94). Occup Environ Med 2002;59:505-11.

22 Medina-Ramon M, Zock JP, Kogevinas $M$, et al. Asthma symptoms in women employed in domestic cleaning: a community based study. Thorax 2003:58:950-4.

23 Norback D, Bjornsson E, Janson C, et al. Asthmatic symptoms and volatile organic compounds, formaldehyde, and carbon dioxide in dwellings. Occup Environ Med 1995;52:388-95

24 Smedje G, Norback D, Edling C. Asthma among secondary schoolchildren in relation to school environment. Clin Exp Allergy 1997;27:1270-8.

25 Millqvist $E$, Bengtsson $U$, Lowhagen $O$. Provocations with perfume in the eyes induce airway symptoms in patients with sensory hyper-reactivity. Allergy 1999;54:495-9.

26 Ross DJ, Keynes HL, McDonald JC. SWORD '97: surveillance of work-related and occupational respiratory disease in the UK. Occup Med 1998;48:481-5.

27 ALSPAC. http://www.ich.bris.ac.uk/alspac.html, 2001.

28 Golding J, Pembrey M, Jones R, the ALSPAC Study Team. ALSPAC-The Avon Longitudinal Study of Parents and Children. I. Study methodology. Paediatr Perinatal Epidemiol 2001;15:74-87.

29 Farrow A, Taylor H, Northstone K, the ALSPAC Study Team, et al. Symptoms of mothers and infants related to total volatile organic compounds in household products. Arch Environ Health 2004 (in press).

30 Venn AJ, Cooper M, Antoniak M, et al. Effects of volatile organic compounds, damp and other environmental exposures in the home on wheezing illness in children. Thorax 2003;58:955-60.

31 Martinez FD, Wright AL, Taussig LM, et al. Asthma and wheezing in the first six years of life. N Engl J Med 1995;332:133-8.

32 Stick SM, Burton PR, Gurrin L, et al. Effects of maternal smoking during pregnancy and a family history of asthma on respiratory function in newborn infants. Lancet 1996;348:1060-4.

33 Dezateux C, Stocks J, Dundas I, et al. Impaired airway function and wheezing in infancy: the influence of maternal smoking and a genetic predisposition to asthma. Am J Respir Crit Care Med 1999;159:403-10.

34 Sherriff A, Peters TJ, Henderson AJ, Strachan D, the ALSPAC Study Team. Factors associated with patterns of wheeze in infants followed longitudinally from birth to $3 \frac{1}{2}$ years. Int J Epidemiol 2001;30:1473-84.

\section{LUNG ALERT}

\section{A novel approach to vaccination for common respiratory pathogens}

$\Delta$ Goldenberg HB, McCool TL, Weiser JN. Cross-reactivity of human immunoglobulin G2 recognizing phosphorylcholine and evidence for protection against major bacterial pathogens of the human respiratory tract. J Infect Dis 2004; 190:125463

n this study phosphorylcholine (ChoP), an antigenic component found on the cell surface of major bacterial pathogens, is investigated as a potential target for a putative single vaccine with activity against multiple respiratory pathogens and, in particular, Streptococcus pneumoniae and Haemophilus influenzae. A number of experiments were performed using human ChoP-specific antibody purified from pooled serum gamma globulin. Initially, purified ChoP antibody was shown to recognise both $\mathrm{ChoP}^{+} \mathrm{H}$ influenzae and pneumococcal lipoteichoic acid. Subsequently it was shown that the antibody was primarily of the IgG2 subtype. The authors then went on to perform in vitro killing assays and demonstrated effectiveness against some clinical isolates of non-typable $H$ influenzae and some serotypes of $S$ pneumoniae. Finally, passive immunisation using intraperitoneal injection of human antiChoP antibody into mice, followed by intraperitoneal pneumococcal challenge (of the less virulent transparent type), conferred $100 \%$ survival on the mice.

Strategies of immunisation designed to increase levels of anti-ChoP IgG2 antibody may enhance host defences against many major bacterial infections of the respiratory tract. This results in a novel vaccine approach-namely, one vaccine for multiple pathogens.

J Pickles

British Lung Foundation Research Fellow, King's College Hospital, London, UK; joanna.pickles@kcl.ac.uk 